\title{
TRATAMIENTO DE LA INFORMACIÓN CIENTÍFICA EN LAS EDICIONES DIGITALES DE LOS PERIÓDICOS ESPAÑOLES
}

\author{
The treatment of scientific information in the digital \\ editions of Spanish newspapers
}

Lourdes López-Pérez y María-Dolores Olvera-Lobo
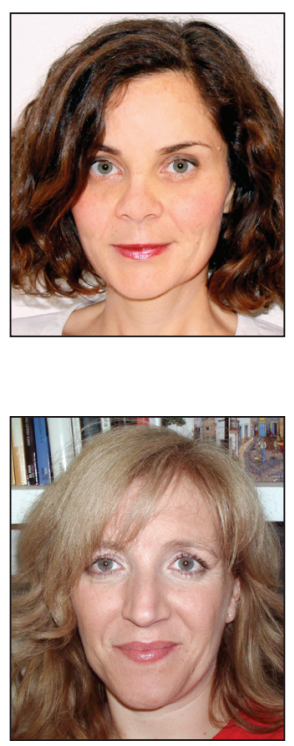

Lourdes López-Pérez es licenciada en ciencias de la comunicación y máster en información y comunicación científica por la Universidad de Granada. Ha desarrollado su carrera profesional en el ámbito de la comunicación científica tanto en medios de comunicación, como centros de investigación y museos y ha ejercido como docente de redacción periodística en la Escuela de Protocolo de Granada. Forma parte del grupo de investigación Acceso y evaluación de la información científica de la Universidad de Granada y es coautora de varios artículos publicados en revistas especializadas nacionales e internacionales.

http://orcid.org/0000-0002-5787-1531

lourdeslopez@correo.ugr.es

María-Dolores Olvera-Lobo es doctora en documentación, profesora titular del Departamento de Información y Comunicación de la Universidad de Granada y docente en las Facultades de Comunicación y Documentación y de Traducción e Interpretación. Ha impartido cursos en programas de doctorado, de máster y de posgrado en diversas universidades. Ha liderado grupos, proyectos de investigación y proyectos de innovación docente. Es miembro del Grupo SCImago, unidad asociada del CSIC. Es autora y/o coautora de libros, capítulos y de decenas de artículos publicados en revistas especializadas nacionales e internacionales.

http://orcid.org/0000-0002-0489-7674

molvera@ugr.es

Universidad de Granada, Departamento de Información y Comunicación Colegio Máximo, Campus Cartuja, 18071 Granada, España

\section{Resumen}

Se analiza el tratamiento de la información científica en las ediciones digitales de los periódicos de mayor audiencia en España, El país, El mundo, $A B C$ y 20 minutos, en diciembre de 2012, 2013 y 2014. El objeto de estudio son las noticias publicadas, de las que se ha determinado su número, se han identificado las fuentes de información utilizadas y el tema tratado, en lo que a área científica se refiere. Los datos obtenidos concluyen que las ediciones online, más allá de superar los handicaps de homogeneización y selectividad presentes en la cobertura en la prensa escrita, lo han acentuado. En los cuatro medios la biología y la biomedicina, así como a la astrofísica, son las áreas más mediáticas. Además, recurren fundamentalmente a dos tipos de fuentes, las revistas de alto impacto y los centros de investigación, en su mayoría de procedencia internacional.

\section{Palabras clave}

Periodismo científico; Revistas de impacto; Fuentes de información; Ciencia; Periodismo digital; Comunicación pública de la ciencia.

\footnotetext{
Abstract

The treatment of scientific information is analysed in the digital edition of the most-read newspapers in Spain: El país, EI mundo, $A B C$ and 20 minutos in December 2012, 2013 and 2014. The subject of inquiry is the published news, of which the quantity has been determined, the sources identified, and each issue categorized by the area of science to which it refers. The data obtained leads to the conclusion that the online editions, rather than overcoming the disadvantages of homogenisation and selectivity, have accentuated them. Biology and biomedicine, along with astrophysics, are the most high-profile areas. Furthermore, they rely fundamentally on two types of source: high-impact journals and research centres, most of them international.
} 


\section{Keywords}

Science journalism; Impact journals; Information sources; Science; Digital journalism; Public communication of science.

López-Pérez, Lourdes; Olvera-Lobo, María-Dolores (2015). "Tratamiento de la información científica en las ediciones digitales de los periódicos españoles". El profesional de la información, v. 24, n. 6, pp. 766-777.

http://dx.doi.org/10.3145/epi.2015.nov.08

\section{Introducción}

La homogeneización en la cobertura de la información científica en la prensa escrita ha sido un tema ampliamente abordado por la literatura especializada (Hansen, 1994; Bourdieu, 2003; De-Semir; Ribas; Revuelta, 1998; De-Semir, 2000; Stryker, 2002; Russell, 2008). Algunos autores han encontrado las causas de esta falta de pluralidad en la prominencia de las revistas de impacto y de las instituciones gubernamentales, así como de la industria, como principales fuentes de información (Entwistle, 1995; Ribas, 1998; Hotz, 2002; Elías, 2002a, 2002b; Goirena; Garea, 2002; Van-Trigt et al., 1994; Weitkamp, 2003; Fernández-Muerza, 2005; Veneu; Amorim; Massarani, 2008; Williams; Clifford, 2009; González-Alcaide; Valderrama-Zurián; Aleixandre-Benavent, 2009). Un protagonismo que, en el caso de las publicaciones científicas especializadas, ha provocado además una tendencia a la internacionalización de las noticias, ya que la mayor parte de los resultados que se publican en las mismas son de origen inglés o norteamericano (Einsiedel, 1992; Bucchi; Mazzolini, 2003; Fernández-Muerza, 2005).

\section{Sólo un tercio de las noticias científicas publicadas hace referencia a centros de investigación españoles}

Otro de los aspectos destacados por los académicos en cuanto al análisis de la cobertura de ciencia en prensa escrita es la selectividad, referida a la priorización de unos temas sobre otros. La mayoría de los trabajos apuntan a la medicina y la salud como el área protagonista, e incluso hablan de "medicalización de las noticias" científicas (Einsiedel, 1992; Jerome, 1986; Pellechia, 1997; Bauer, 1998; Bucchi; Mazzolini, 2003; Weitkamp, 2003).

Hasta qué punto se mantiene esta tendencia conocida como "periodismo científico alimentado con cuchara" (Russell, 2008) en las ediciones digitales de los periódicos, y cómo está afectando a la imagen que se proyecta de la ciencia española son aspectos escasamente abordados hasta el momento. En trabajos anteriores (Olvera-Lobo; López-Pérez, 2013; 2014; López-Pérez; Olvera-Lobo, 2015) se ha apuntado la tendencia a la homogeneización de la información y a la selectividad temática en las ediciones online.

En el estudio que se presenta se han incluido tres períodos de análisis que demuestran la consolidación de esta tendencia de forma general en las ediciones digitales de los cuatro periódicos de mayor audiencia El país, El mundo, $A B C$ y 20 minutos (Comscore, 2012; 2013; 2014). La internacionalización de la información, que representa más de un $70 \%$ de las noticias publicadas, y la prevalencia de revistas de alto impacto como Nature y PNAS se repite en los cuatro medios de comunicación. Sin embargo, en 2014 se muestran ciertas diferencias con mayor protagonismo de la información nacional en El país con respecto a los otros tres medios estudiados. Este cambio puede estar influido, tal y como explicamos en las conclusiones, por la creación de una sección específica de ciencia en su edición digital durante ese año.

\section{El impacto de internet en el periodismo científico}

La cobertura de la ciencia en las ediciones digitales de los medios de comunicación aún no es un área muy estudiada por los académicos e investigadores de la comunicación pública de la ciencia. Esta ausencia de trabajos ha sido reivindicada por autores como Brossard y Scheufele (2013) quienes consideran urgente el análisis del tratamiento que los medios tradicionales están dando a la ciencia en sus ediciones en internet.

El cambio del acceso a las fuentes de información es uno de los temas que concentran más interés (Curtin; Rhodenbaugh, 2001; Gans, 2010; Hu; Sundar, 2010; Granado, 2011). En un análisis basado en entrevistas a periodistas científicos (Granado, 2011) éstos afirmaban que internet ha facilitado el contacto directo con los científicos, la ampliación de la información y el acceso a documentos que antes no estaban disponibles, pero también ha incrementado la dependencia de las revistas de impacto y de las notas de prensa de los centros de investigación. La información científica es ahora menos diversa y más homogénea que antes debido a la concentración de los periodistas científicos en unas cuantas fuentes.

\section{Nature, Science y PNAS son las tres re- vistas con mayor protagonismo en las ediciones digitales españolas}

Según Kiernan (2000) las revistas de impacto ganan protagonismo en las ediciones digitales. Algo que también parece estar afectando en otro sentido, es decir, en el protagonismo de la procedencia geográfica inglesa y norteamericana de los hechos noticiables referenciados. Sobre esta idea se ahonda en el White paper on a European communication policy (Commission of the European Communities, 2006) donde se afirma que la cobertura de la ciencia europea es muy limitada. $Y$ es que las revistas de impacto concentran una gran mayoría de artículos procedentes de centros e instituciones de estas nacionalidades y éstas son, a su vez, uno de los principales recursos de los medios de comunicación, con lo que se produce una tendencia circular según la cual la ciencia producida fuera del circuito de las publicaciones de impacto más reconocidas no tiene cabida en la agenda informativa. 


\section{Una aproximación a la investigación sobre la cobertura de la información científica en España}

Las fuentes de información y el análisis formal del discurso periodístico son los dos aspectos más tratados en los estudios sobre periodismo científico firmados por autores españoles. Al igual que en el ámbito internacional el recurso permanente a las revistas de impacto como fuentes de información primaria han centrado los principales trabajos empíricos publicados en nuestro país (Ribas, 1998; DeSemir, 2000; Elías, 2002a; 2002b; Goirena; Garea, 2002; Fernández-Muerza, 2005; Pont-Sorribes, Cortiñas-Rovira; Di-Bonito, 2013).

La mayoría de los autores coinciden en la inclinación generalizada que se observa en los medios de comunicación españoles a la homogeneización de la información científica. También se habla de mimetismo (De-Semir, 2000) o de circulación circular de la información (Bourdieu, 2003) en el sentido de que unos medios cubren lo mismo que la competencia y todos a su vez prestan atención a los referentes internacionales en prensa como pueden ser The New York times, The Times o Le Monde, entre otros.

La similitud en la selección de los temas es producto, en gran parte, de la gran dependencia actual del periodismo científico español respecto de las publicaciones especializadas.

Nature y Science se presentan como las fuentes más recurrentes para los principales periódicos impresos españoles (Ribas, 1998; De-Semir, 2000; Goirena; Garea, 2002; Elías, 2002a, 2002b; Fernández-Muerza, 2005; González-Alcaide; Valderrama-Zurián; Aleixandre-Benavent, 2009; PérezBahón, 2010). Esta inclinación por el periodismo perezoso (De-Semir, 2013) deja en manos de dos o tres revistas científicas la selección de la información científica noticiable. Son las principales gestoras del fenómeno de agenda setting en los medios de comunicación, por el que éstas imponen a los ciudadanos aquellos hechos que son noticiables (Elías, 2002a).

De este modo, en el caso del periodismo científico la información es sometida a un doble examen de noticiabilidad. El primero es el que realizan las revistas de alto impacto y, sobre éste, una segunda selección temática corresponde a los periodistas y editores de los medios. Esto provoca una monopolización del periodismo científico (Ribas, 1998; González-Alcaide; Valderrama-Zurián; Aleixandre-Benavent, 2009) y fomenta unos claros circuitos informativos tanto geográficos como científicos (Fernández-Muerza, 2005). En el primer caso, por la preponderancia del mundo anglosajón, especialmente de EUA, y en el segundo caso, por la importancia que se le da a los investigadores en función del país en el que trabajan -y, una vez más, vuelven a tener más protagonismo los centros de procedencia anglosajona que los nacionales-.

Además, dentro de la procedencia nacional, vuelve a haber prevalencia de unos puntos geográficos sobre otros. Se produce así lo que Elías (2002c) ha definido como periodismo centralista, en el sentido de que sólo cuenta el estado de opinión de los investigadores de la capital del país. Ya que la mayor parte de los redactores especializados trabajan en ediciones nacionales y, ya sea por comodidad o por dificultad de acceso, acuden a centros de investigación y científicos ubicados en Madrid.

La irrupción de internet en el periodismo científico parece no haber modificado esta dependencia de las revistas científicas, sino más bien al contrario, ha acrecentado la situación (Pérez-Bahón, 2010). El uso de la Red ha permitido ganar tiempo a los periodistas especializados que no deben salir a la calle a buscar sus fuentes. Una nueva realidad que han aprovechado las revistas de alto impacto realizando envíos digitales semanales de notas de prensa con artículos científicos ya "traducidos" al lenguaje periodístico.

Internet se ha convertido en un factor de reactivación para que ciertos generadores de información interesada potencien su penetración en las redacciones. Una uniformidad que no sólo se manifiesta en lo que a fuentes de información se refiere, sino también en los temas tratados. La medicina (Elías, 2002c; Aranes-Usandizaga; Landa-Montenegro, 2002; Goirena; Garea, 2002; González-Alcaide; ValderramaZurián; Aleixandre-Benavent, 2009; Pérez-Bahón, 2010), el medio ambiente (Aranes; Landa, 2002; Goirena; Garea, 2002; González-Alcaide; Valderrama-Zurián; AleixandreBenavent, 2009) y la arqueología (González-Alcaide; Valderrama-Zurián; Aleixandre-Benavent, 2009) son los temas más recurrentes en las noticias científicas publicadas por los principales periódicos impresos españoles y es importante conocer si esta selectividad temática también se repite en las ediciones digitales..

Los resultados apuntan a una 'medicalización' y 'astronomización' de la información científica española en las versiones online de los periódicos

\section{Objetivos y metodología}

El objetivo de este trabajo es analizar la cobertura de la información científica en las ediciones digitales de los periódicos españoles. La idea de centrarnos en ediciones digitales responde principalmente al hecho de que internet es la principal fuente de información científica para el $85 \%$ de los jóvenes de 15 a 24 años y la segunda para la población general, muy cerca de la televisión (Fecyt, 2013). Por tanto, conocer la imagen que están proyectando de la ciencia a la sociedad es de gran relevancia. El estudio se concentra en tres aspectos fundamentales, la cuantificación de las noticias publicadas, la identificación de las fuentes de información utilizadas y la determinación de las áreas científicas de las noticias.

La muestra de estudio se ha establecido atendiendo al valor de audiencia. Así, se han seleccionado los cuatro con más lectores (Comscore, 2012): El país, El mundo, ABC y 20 minutos.

Los cuatro medios analizados dedican secciones específicas a las noticias científicas. Las ediciones digitales de El mundo, $E l$ país y $A B C$ coinciden en la denominación de la sección, 
a saber, Ciencia. Si bien en los dos primeros, es visible en la homepage, en El país está integrada como una subsección del área de Sociedad hasta octubre de 2014, cuando se crea una sección propia dedicada a ciencia. 20 minutos no tiene una sección de ciencia como tal hasta marzo de 2014; previamente los temas científicos estaban integrados en dos secciones, medio ambiente y salud. El hecho de que los temas se engloben exclusivamente bajo estas dos áreas influye en los resultados finales en dos niveles. En primer lugar, porque habrá temas científicos que se hayan publicado en otras secciones que han quedado fuera del análisis, y, en segundo lugar, por una prevalencia en este medio de los temas mencionados, medio ambiente y salud.

Se han definido como noticias científicas aquellas que incluyen resultados de investigaciones en todas las áreas de conocimiento o abordan resultados relacionados con las ciencias aplicadas, la ingeniería o la medicina (Bucchi; Mazzolini, 2003). También se han considerado noticias científicas los artículos donde la ciencia o su metodología son la base de argumentación.

La metodología desarrollada para la evaluación ha consistido en el diseño de una checklist ad hoc estructurada en torno a tres dimensiones de análisis, cuantificación de la cobertura, homogeneización y selectividad (tabla 1). Los items se han definido en función de los objetivos específicos del estudio y a partir de la revisión bibliográfica.

La selección de áreas de investigación incluida en el análisis de la selectividad responde a la propia estructura de áreas científicas que establece el Consejo Superior de Investigaciones Científicas (CSIC, 2012). El criterio al seguir el mismo establecido por el CSIC responde por un lado a que es el mayor organismo público de investigación en España y, por el otro, a la falta de normalización académica a la hora de establecer y definir las áreas de investigación.
Tabla 1. Checklist ad hoc para análisis de los medios de comunicación

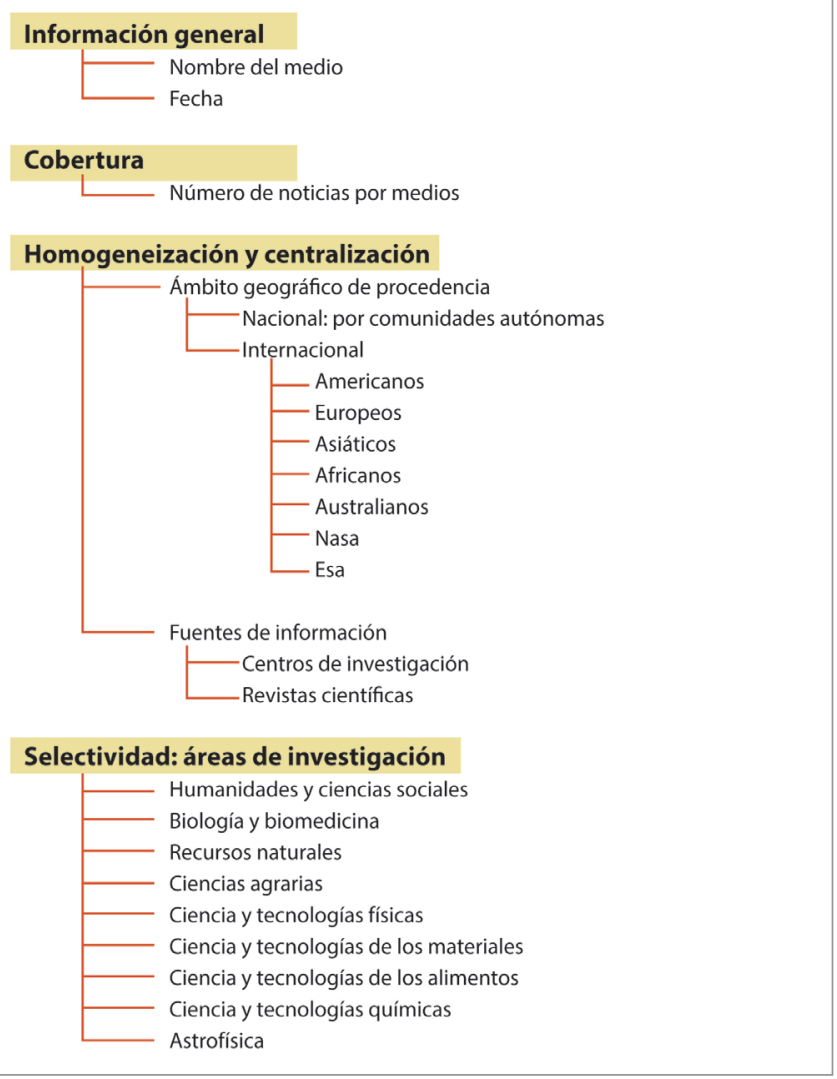

este último año. 2014 es el año en el que este periódico ha llevado a cabo una mayor cobertura de hechos relacionados con el desarrollo científico, algo que tal vez esté relacionado con la creación de una sección específica de ciencia en octubre de 2014, hasta ese momento Ciencia era una subsección integrada en Sociedad (gráfico 1).
Así, las áreas observadas han sido humanidades y ciencias sociales, biología y biomedicina, recursos naturales y medio ambiente, ciencias agrarias, ciencia y tecnologías físicas, ciencia y tecnologías de los materiales, ciencia y tecnologías de los alimentos, ciencia y tecnologías químicas, y astrofísica.

El período de estudio ha sido del 1 al 31 de diciembre de 2012, 2013 y 2014, con la finalidad de observar la evolución experimentada en el tiempo.

\section{Resultados}

\subsection{El país}

Uno de los resultados más destacables de El país en el período analizado es el incremento de la cobertura de la información científica. Desde el año 2012 al 2014 las noticias científicas publicadas en este periódico han aumentado en un $131 \%$. Una tendencia que ya se experimentó entre el año 2012 y 2013, con un 30,3\% más de noticias, y de 2013 a 2014, con un 74,4\% más

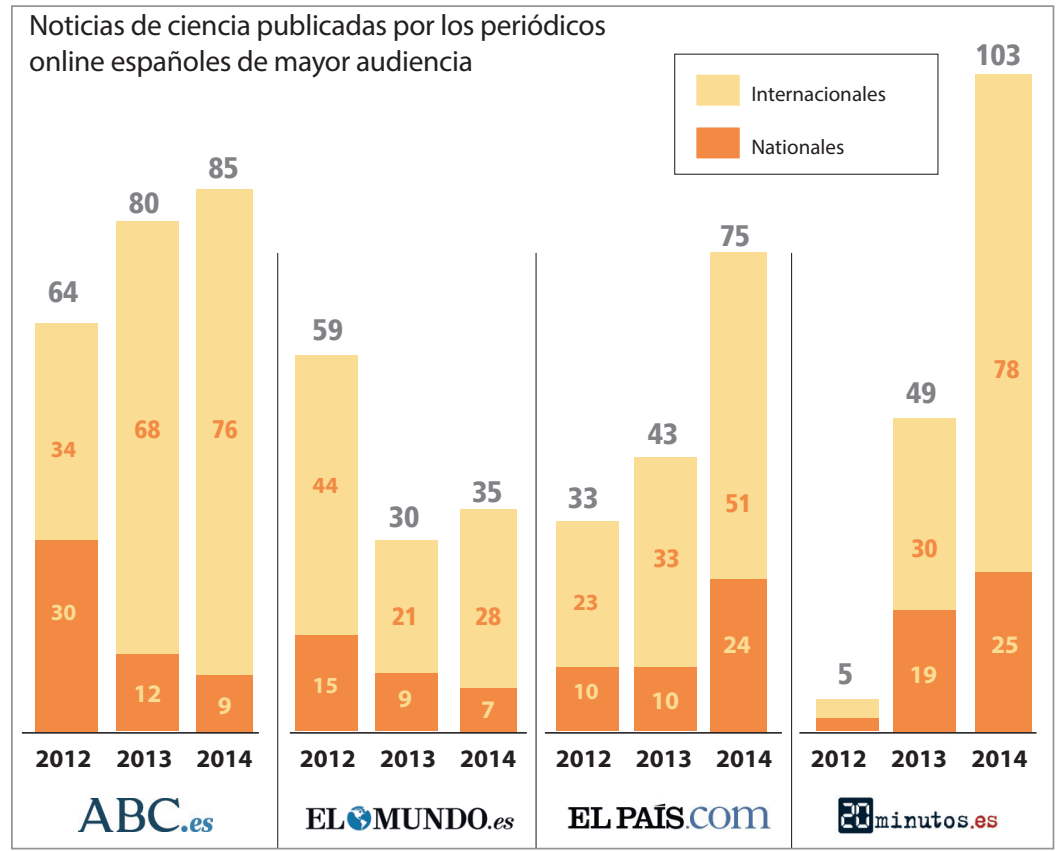

Gráfico 1. Noticias de ciencia publicadas por los periódicos online españoles de mayor audiencia. 
La procedencia geográfica de las fuentes referenciadas en la noticia parece mantenerse a lo largo de los años con un protagonismo significativo del origen internacional, que en los tres años conforma en torno al $71 \%$ del total frente al 30\% aproximadamente de nacional. Esta tendencia señala la dependencia en la cobertura de la información científica de las agencias informativas que, a su vez, dependen de las notas de prensa y comunicados enviados por importantes centros de investigación, ubicados en su mayoría en Norteamérica, y revistas de alto impacto de procedencia igualmente norteamericana e inglesa.

Casi un $10 \%$ de las informaciones publicadas no mencionan una fuente de información específica

El análisis detallado de la procedencia internacional arroja resultados dispares de un año a otro con lo que no se puede establecer una tendencia clara. Así, como se observa en el gráfico 2, en 2012 es llamativo el alto porcentaje de noticias (30,4\%) que hacen referencia a la NASA seguida de las referidas a centros europeos (26\%) y americanos, que alcanzan el $21,7 \%$. Como mencionábamos anteriormente en la influencia informativa de la NASA en la cobertura científica en medios españoles está incidiendo la importante estrategia de comunicación que este organismo realiza para alcanzar impacto mediático.

Otro dato llamativo de este año es el hecho de que el $13 \%$ de las noticias no mencionan una fuente informativa específica, sino más bien hacen una referencia general y/o indirecta como "científicos americanos", "investigadores internacionales", etc.

En el año 2013 hay un gran protagonismo de la investigación de procedencia norteamericana, que representa un $45,5 \%$ frente al 15,1\% europeo, y la NASA vuelve a tener presencia aunque menor $(15,1 \%)$, similar a la otorgada a la Agencia Espacial Europea (ESA), que alcanza el 12,2\%. Este año el número de noticias que no mencionan la fuente de los datos es muy inferior, un $3 \%$.

Sin embargo, en 2014 se invierte esta situación y son los centros europeos los que más aparecen en las noticias publicadas, un $41,4 \%$ frente al $27,5 \%$ de procedencia norteamericana. Las agencias espaciales, tanto la europea con un $5,9 \%$ como la americana con un $7,8 \%$, tienen menos relevancia informativa, y las noticias que no mencionan fuente informativa específica vuelven a superar el $10 \%$, suponiendo en este caso el $11,8 \%$ del total.

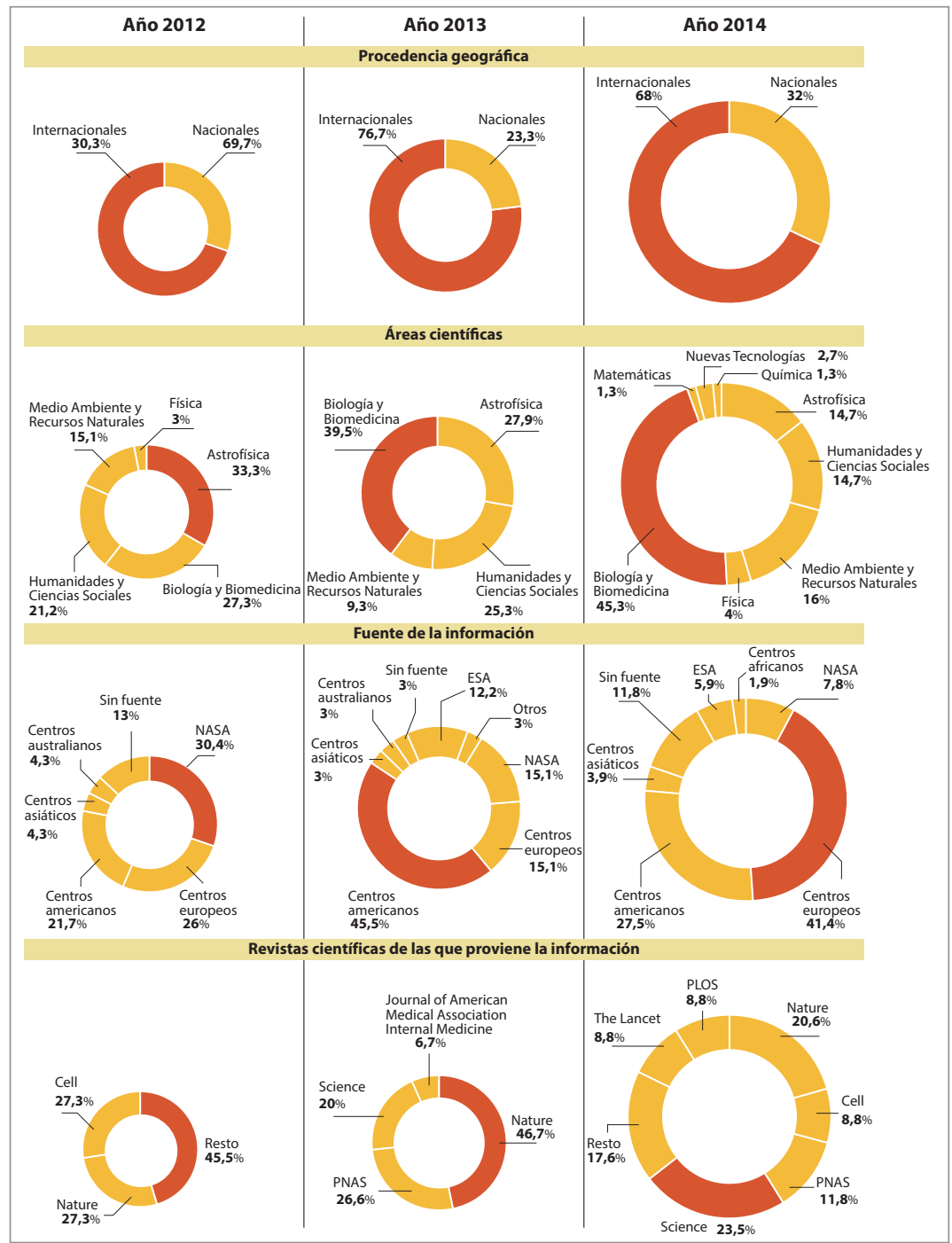

Gráfico 2. Evolución de noticias publicadas en el periódico El país.
Las áreas de investigación con mayor presencia son coincidentes los tres años: astrofísica, biología y biomedicina, y humanidades y ciencias sociales. La astrofísica y la bio-medicina se disputan el primer puesto de un año a otro como vemos en el gráfico 2 . El fuerte protagonismo de la NASA está influyendo en preponderancia de esta área sobre otras. En cuanto a la biología y la biomedicina, son disciplinas muy mediáticas por el alto interés que despiertan. En cuanto a la presencia de las revistas de impacto en las informaciones, tanto en 2012 como en 2013, constituyen en torno al 33\% del total, una cifra que se incrementa más de 10 de puntos en 2014, representando el 45,3\%.

Nature es la que copa el mayor el volumen de información en el año 2012 $(27,3 \%)$ y en el año 2013 (46,7\%), mientras que en 2014 es Science la que tiene mayor relevancia con un 23,5\%. Las tres revistas más referenciadas en los tres años son Nature, Science y PNAS (Proceedings of the National Academy of Sciences of the United States of America). Una vez más tanto el alto porcentaje de referencias a las revistas de alto impacto como fuentes de información, como la presencia de Nature y Science, dos revistas con sólidos departamentos de comunicación y que dan gran importancia al impacto de sus trabajos en los medios, hacen inferir la prevalencia de un periodismo "alimen- 
tado con cuchara". Es decir, dependiente de agencias de información y de gabinetes de prensa de instituciones científicas y de revistas de alto impacto.

\subsection{El mundo}

En el caso de El mundo se da una situación inversa a El país en lo que a cobertura de información científica se refiere. Hay un notable descenso de 2012 a 2013 con un $49,2 \%$ de noticias menos de un año a otro, si bien este volumen se mantiene más o menos en 2014 en el que se publica un $40,67 \%$ menos con respecto a 2012 y sólo un 16,6 \% más que en 2013 (gráfico 1). Este descenso puede estar provocado por los recortes de personal en secciones especializadas como la de Ciencia que ha sufrido este periódico en los últimos años.

La procedencia geográfica de las fuentes se mantiene de un año a otro. En torno al 30\% de las noticias hacen referencia a centros nacionales y el $70 \%$ a internacionales. Hay una ligera modificación en 2014, cuando las nacionales representan el $20 \%$ y las internacionales el $80 \%$ como muestra el gráfico 3.

Los centros americanos son protagonistas de la información internacional, representando un 33\% en 2012, un 33,3\% en 2013 y un $39,3 \%$ en 2014 . Le siguen los europeos con un $31,1 \%$ en 2012 , un $19 \%$ en 2013 y un $14,3 \%$ en 2014 .

Al igual que en el caso de El país, la NASA y la ESA tienen una fuerte presencia en las noticias publicadas. La repercusión de la NASA es mayor a la de la ESA para el año 2012, 11,1\% de la primera frente al $8,9 \%$ de la segunda, y para el año $2013,19 \%$ para la NASA y $9 \%$ para la ESA. En 2014 se invierte la situación y la ESA aparece en un $17,8 \%$ de las informaciones frente a un $14,3 \%$ destinado a la NASA.

La referencia a fuentes de información no específicas también se da en el periódico El mundo, utilizando recursos como "científicos americanos", "investigadores europeos", etc. En 2012 un 11,1\% de las informaciones hacen alusión a fuentes no específicas, una cifra que desciende en 2013 a un $4,7 \%$ y vuelve a incrementarse en 2014 , con un $7,1 \%$.

En cuanto a la referencia a noticias científicas en el año 2012, un $51,7 \%$ de las noticias hacen alusión a una revista de impacto, un 40\% en 2013 y un 37,1\% en 2014.

Las revistas Nature y Science vuelven a ser las que tienen mayor protagonismo. En 2012 Nature y New England journal of medicine son las revistas más citadas con $9,7 \%$ cada una. Una situación que cambia en 2013 donde Nature tiene un $41,7 \%$, seguida de PNAS con un $25 \%$.

En 2014 Science le roba el primer puesto a Nature con un
$30,8 \%$ de las referencias, seguida de Nature que está presente en un $23 \%$ de las noticias.

Con respecto a las áreas de investigación hay cambios significativos entre los años 2012, 2013 y 2014. En el primero, la biología y la biomedicina es la gran protagonista representada en un $53,3 \%$ de las noticias, una cifra que desciende sorprendentemente a un $10 \%$ en 2013 y a un 11,4\% en 2014.

La astrofísica ocupa el primer puesto en el año 2013, concentrando un $40 \%$ de las informaciones y en 2014 con presencia en un $34,3 \%$ de las noticias. Las humanidades y ciencias sociales vuelven a ser la tercera área más destacada con un $11,6 \%$ en 2012, un 20\% en 2013 y un 22,9\% en 2014.

Al igual que en el caso de El país los datos apuntan a la idea de consolidación del periodismo "alimentado con cuchara". Por otro lado, el hecho de que el análisis en ambos periódicos sea semejante señala también la tendencia a la homogeneización de la información y refuerza el hecho de que la información científica que se publica en los medios españoles depende de los departamentos de comunicación de los principales centros de investigación internacionales y de las estrategias de difusión de las revistas de alto impacto. 


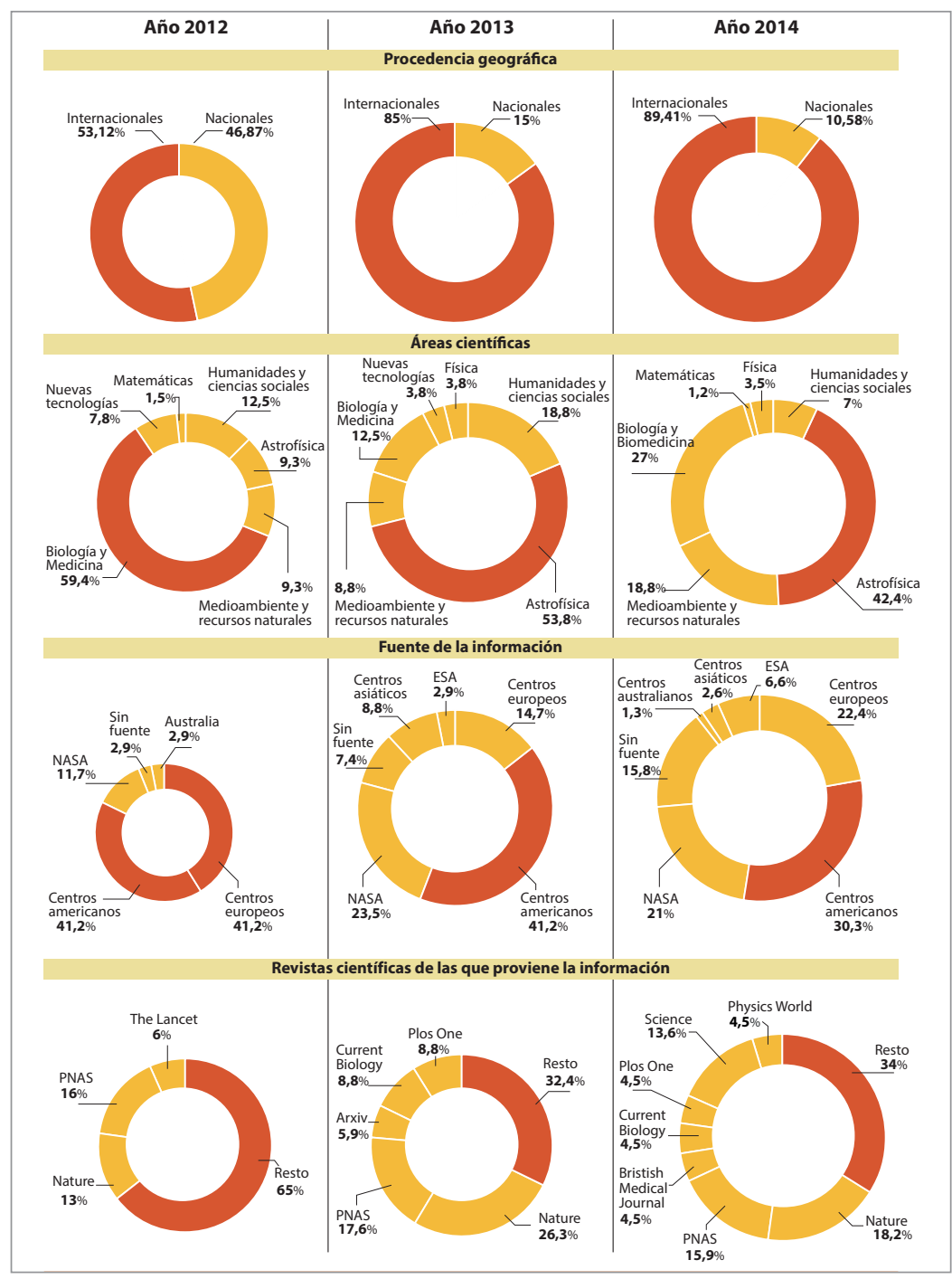

Gráfico 4. Evolución de noticias publicadas en el periódico $A B C$. de las informaciones no señala una fuente específica. La brecha entre procedencia norteamericana y europea se hace muy notable en 2013 con un 41,2\% de referencias a centros americanos y un $14,7 \%$ a europeos. La NASA gana protagonismo ese año y es la fuente del $23,5 \%$ de las informaciones, frente a un escaso $2,9 \%$ en el que la ESA es protagonista. Un $7,4 \%$ de las noticias no refiere fuente específica.

En 2014 los centros americanos siguen siendo protagonistas, pero las diferencias vuelven a acortarse. De este modo, el $30,3 \%$ de las noticias menciona un centro o institución norteamericana y el $22,4 \%$ hace referencia a uno europeo. La NASA sigue teniendo una importante relevancia con presencia en el $21 \%$ de las noticias frente al escaso $6,6 \%$ de la ESA. Un $15,8 \%$ de las noticias no refiere fuente específica.

Las áreas más noticiables son, como en los casos anteriores, la astrofísica, la biomedicina, y las humanidades y ciencias sociales. Es llamativa la variación que existe de la cobertura de información de biomedicina del año 2012 , con un $59,4 \%$ de las noticias al 2013 , con un $12,5 \%$ y al 2014 donde representa el $27 \%$ de las informaciones. En el caso de la astrofísica el cambio es inverso, pasando de un $9,3 \%$ en 2012 , al $53,8 \%$ de 2013 y al $42,4 \%$ de 2014 . La cobertura de hechos informativos sobre humanidades y ciencias sociales también oscila desde un $12,5 \%$ en 2012 , al $18,8 \%$ de 2013 para acabar en un 7\% en 2014.

\section{3. $A B C$}

La cobertura de información científica en la edición digital del periódico $A B C$ experimenta un incremento del $25 \%$ entre los años 2012 y 2013. En 2014 se mantiene la tendencia creciente con un $32,8 \%$ más que en el año 2012 y un $6,3 \%$ más que en 2013 (gráfico 1).

El ámbito de procedencia de las noticias experimenta una notable variación en el período analizado. Así, en 2012 hay un equilibrio entre la información que hace referencia a investigaciones nacionales, un $46,9 \%$, y las que mencionan fuentes internacionales, el 53,1\%. La distancia entre ambos valores aumenta en 2013 , cuando el $15 \%$ de las noticias menciona una fuente nacional y el $85 \%$ una internacional. En 2014 se acrecienta aún más la diferencia entre la referencia nacional de las noticias, con un $10,6 \%$, y la internacional, con el $89,4 \%$.

Los centros de investigación americanos son los que tienen un mayor protagonismo durante los tres años del período analizado, seguidos de los europeos y de centros singulares como la NASA, tal y como se observa en el gráfico 4. En 2012 el 41,2\% de las noticias proviene de instituciones norteamericanas y otro tanto de las europeas. La NASA es la fuente informativa del $11,7 \%$ de las noticias y un $2,9 \%$
Las revistas de alto impacto tienen un importante protagonismo en la edición digital del periódico $A B C$. En 2012 un $48,4 \%$ de la información tiene como fuente una revista especializada, en 2013 un 42,5\% y en 2014 más de la mitad con un $51,8 \%$. En el caso de $A B C$, Nature y PNAS son las que tienen una mayor presencia en los tres años analizados. Sólo en 2014 irrumpe en el tercer puesto Science con un $13,6 \%$, frente al $18,2 \%$ de Nature y al 15,9 de PNAS.

Una vez más se repite la tendencia señalada en El país y en El mundo.

\subsection{0 minutos}

La edición online del periódico 20 minutos es la que experimenta un incremento más sorprendente del año 2012 al 2013 con un $88 \%$ más de noticias científicas publicadas y al 2014, donde el porcentaje de incremento se eleva hasta el $1.960 \%$ (gráfico 1). Este significativo interés por la información científica puede deberse a la creación en 2014 de una sección específica para la ciencia en la edición digital.

Al igual que en los medios analizados anteriormente, la información con referencias a centros e instituciones internacionales es significativamente superior a la que tiene como fuente un centro nacional en los tres años analizados. En 
2012 y 2013 los porcentajes son similares, de modo que el $40 \%$ hace referencia a medios nacionales y el $60 \%$ a internacionales, una distancia que incrementa en 2014 cuando el 75,7\% de las informaciones mencionan un centro internacional y el $24,3 \%$ nacional.

En los tres años analizados las instituciones de ámbito norteamericano son las que tienen un mayor protagonismo, seguidas muy de cerca por las europeas (gráfico 5). En 2012 y 2013 las cifras son similares. Centros europeos y americanos aparecen en un $33,3 \%$ de las noticias respectivamente en el año 2013 y en un $40 \%$ en ambos casos para el año 2013. En 2014 se amplía levemente la diferencia y el $34,7 \%$ de las noticias tienen como fuente una institución norteamericana frente al $20 \%$ que hace referencia a un centro europeo. La NASA sólo adquiere relevancia en el año 2014 con presencia en un $20 \%$ de la información, mientras que las noticias sin fuente específica representan un 20\% en 2013 y un 16\% en 2014.

Biología y medicina ofrecen los temas estrella en todo el período analizado: en 2012 representan un $60 \%$. El $40 \%$ restante se lo reparten a partes iguales física y humanidades y ciencias sociales. El porcentaje de biomedicina sube a un $67,3 \%$ en 2013 , le siguen medio ambiente con un $24,5 \%$ y a continuación astrofísica y humanidades y ciencias sociales con un 4,1\% cada una. En 2014 a la biomedicina le corresponde el $48,5 \%$ seguida de la astrofísica con un $21,4 \%$ y el medio ambiente y recursos naturales con un $18,4 \%$.

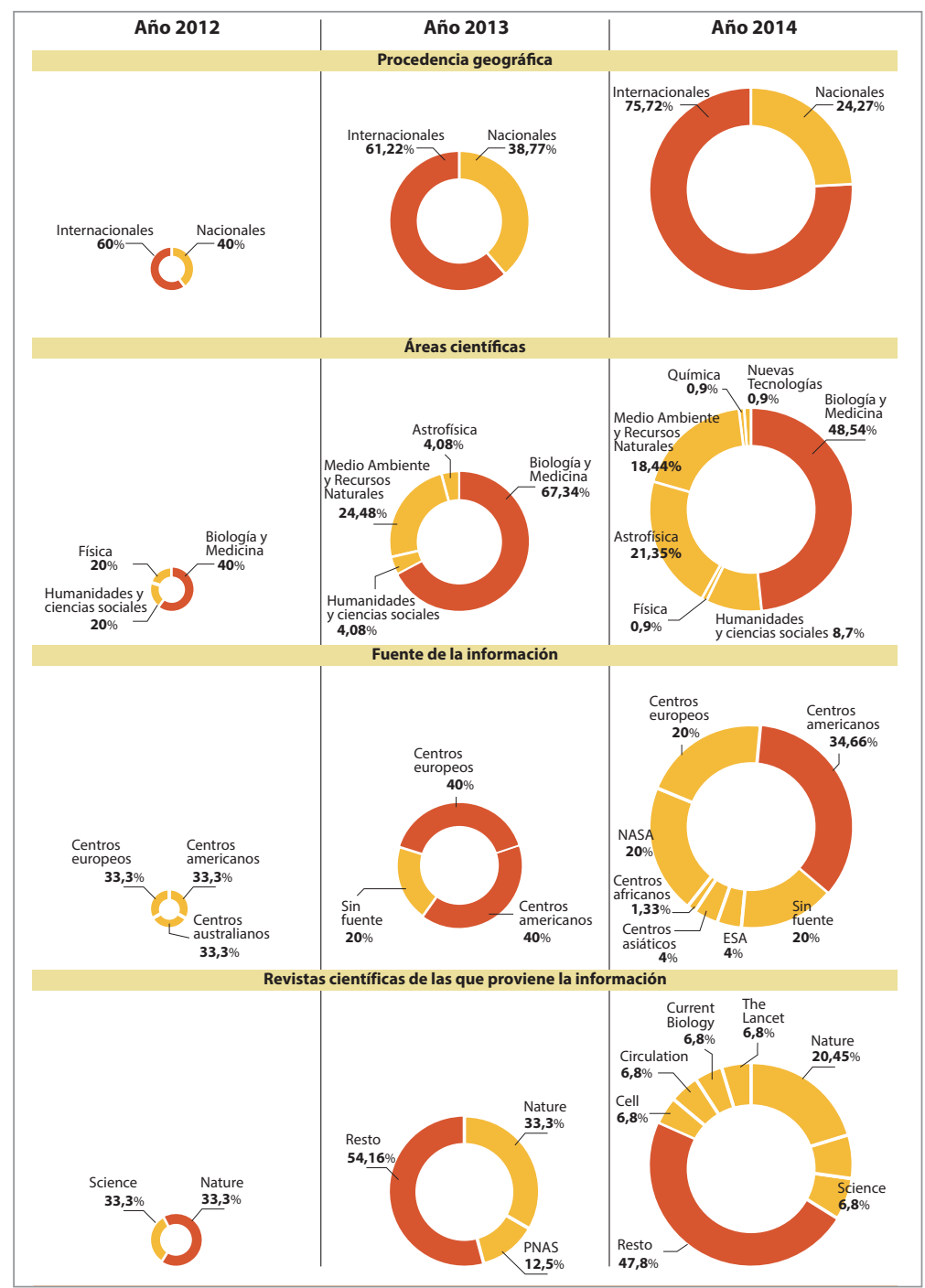

Gráfico 5. Evolución de noticias publicadas en el periódico 20 minutos.
Las revistas de alto impacto tienen un mayor protagonismo en 2012 (referenciadas en el 60\% de las noticias) y en 2013 (en un 49\%), que en 2014 donde aparecen como fuente del $42,7 \%$ de las noticias. Nature, Science y PNAS son las tres revistas más recurrentes en las informaciones publicadas en los tres períodos.

Los resultados obtenidos en el análisis de 20 minutos permiten confirmar la idea que hemos apuntado sobre los medios anteriores y que permite inferir que el tratamiento de la información científica de las ediciones digitales de los medios de comunicación españoles están en manos de las agencias de información y de los departamentos de marketing y comunicación de los organismos internacionales de investigación y de las revistas de alto impacto.

Finalmente, en el gráfico 6 se pueden observar los datos globales por años de procedencia, fuentes de información, áreas de investigación más mediáticas y revistas especializadas más citadas.

\section{Discusión y conclusiones}

Los trabajos científicos realizados por investigadores españoles han perdido protagonismo en los periódicos digitales españoles frente a los desarrollados por centros o instituciones internacionales. Sólo en torno a un tercio de las no- ticias científicas publicadas en medios de comunicación españoles hacen referencia a una fuente de ámbito nacional. En cuanto a la tendencia a la "americanización" de la información científica, que ya apuntaban estudios anteriores en prensa escrita (Einsedel, 1992; Bucchi; Mazzolini, 2003), se mantiene también en las ediciones digitales de los periódicos españoles. En los años 2013 y 2014, más de una tercera parte de las noticias mencionan a una institución científica norteamericana. Estos resultados apuntan a una dependencia de estos medios de las agencias de información y de los departamentos de comunicación de importantes centros de investigación internacionales, la mayoría de ellos localizados en Norteamérica.

También es destacable el fuerte protagonismo que tiene la agencia espacial americana NASA que, en las tres etapas temporales analizadas, es mencionada por aproximadamente un $15 \%$ de las informaciones publicadas. Este protagonismo responde a dos factores, el primero son las fuertes estrategias de comunicación y difusión internacional que realiza esta agencia, y el segundo es el interés mediático y social que despierta la astrofísica. Ambas cosas pueden estar influyendo para que los trabajos de esta institución norteamericana sean más relevantes, informativamente hablando, que otros también de importancia científica de- 


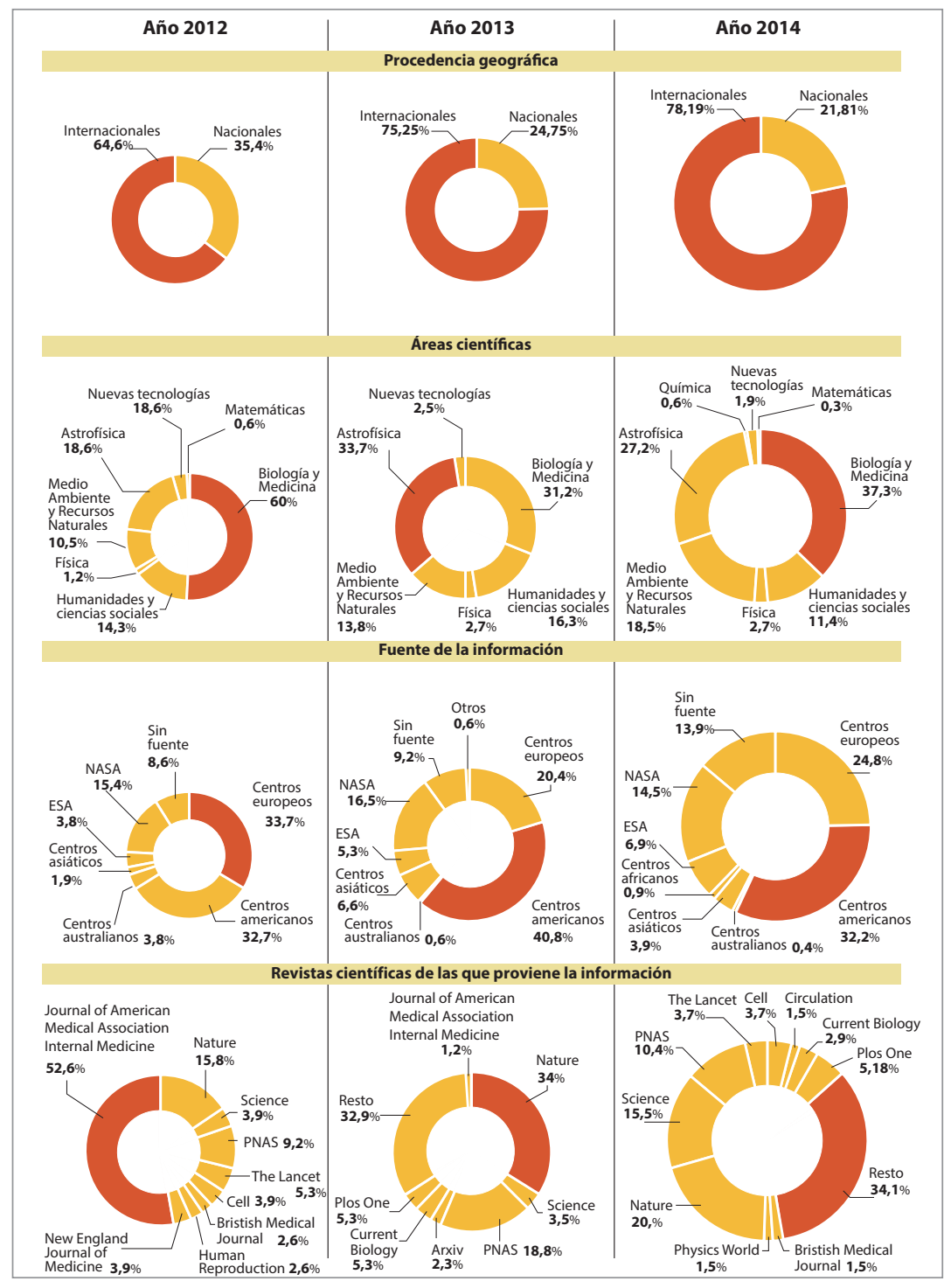

Gráfico 6. Resultados globales de los medios estudiados en los años 2012, 2013 y 2014. dios realizado por la revista El profesional de la información, que sólo consiguió eco en blogs y medios secundarios (CortiñasRovira; Ramon-Vegas, 2013).

Esto hechos vuelven a incidir en la idea de que el tratamiento de la información científica en las ediciones digitales de los medios de mayor audiencia en España depende de las agencias de información y de las notas de prensa enviadas por revistas de alto impacto y centros de investigación. De hecho, la producción propia es cada vez más insignificante, lo cual puede deberse a los recortes de personal en los medios de comunicación, en general, y en las áreas de especialización más concretamente.

La selectividad también es un hecho contrastado en el trabajo que aquí se presenta. E incluso podemos hablar de "medicalización de la información" ya que la biología y la biomedicina son el área con mayor protagonismo los años 2012 y 2014 . El interés social y mediático de estas disciplinas influye sin duda en su protagonismo. A esa "medicalización" le añadimos una "astronomización" de la información, si es que este término pudiera ser admitido, ya que esta disciplina ocupa un lugar prevalente en las noticias científicas españolas, llegando a ser el área protagonista en el año 2013. La fuerte presencia de la NASA como fuente informativa puede explicar esta relevancia que la información sobre astronomía está adquiriendo en los medios españoles.

sarrollados por centros de investigación españoles en este campo como los Institutos de Astrofísica de Canarias y de Andalucía o europeos, como la Agencia Espacial Europea.

El concepto de homogeneización de la información científica en prensa escrita, ya avanzado por algunos de los académicos más eminentes en este campo, se confirma en los resultados obtenidos en este estudio. No sólo por la prevalencia de unas áreas sobre las demás, sino también por el protagonismo de dos revistas en los tres años analizados, Nature y PNAS. En torno al $40 \%$ de las noticias hacen referencia a una revista de alto impacto como fuente y, de ese $40 \%$ estas dos revistas son las que mantienen una presencia mayor, hasta 2014 cuando también se suma Science entre las más relevantes. Nature constituye una de las fuentes más recurrentes en las ediciones digitales al igual que lo era en las versiones impresas de los periódicos (Ribas, 1998; De-Semir, 2000; Goirena; Garea, 2002; Elías, 2002a, 2002b; Fernández-Muerza, 2005; González-Alcaide; Valderrama-Zurián; Aleixandre-Benavent, 2009; Pérez-Bahón, 2010). En este punto es importante señalar que en ningún medio aparece referida una revista española en todo el período analizado. Valga como ejemplo el experimento de difusión a los me-
Asimismo, parece importante señalar el hecho de cómo la creación de secciones de ciencia en El país y 20 minutos durante el año 2014 ha mejorado la cobertura de información científica, no sólo desde el punto de vista cuantitativo, sino también desde el punto de vista cualitativo al incrementar considerablemente, en el caso de El país, las noticias sobre investigaciones desarrolladas en España y Europa.

Por otro lado, es reseñable el hecho de que casi un $10 \%$ de las informaciones no mencionen una fuente de información específica. Algo que resta credibilidad a la información publicada y que denota dejadez en el trabajo periodístico. Se dan por válidos los datos que seguramente han entrado por agencia o por nota de prensa, sin contrastar la información con fuentes autorizadas.

Podríamos concluir que la selectividad y homogeneidad que caracteriza a la información científica publicada en las ediciones digitales de los periódicos españoles de mayor audiencia provoca una representación sesgada de la ciencia española y una infravaloración de la misma al dar protagonismo a la internacional, en ocasiones, por el mero hecho de proceder de fuera de nuestras fronteras. 
De hecho, no sólo no se superan los sesgos en la cobertura presentes en los medios tradicionales, si no que se consolidan. La situación es compleja sobre todo en un momento que el periodismo en general afronta una doble crisis, la profesional y la económica. Esto hace difícil cambiar la práctica de "periodismo alimentado con cuchara". La falta de recursos humanos y de especialización perjudica a la cobertura de la información científica en este caso y otorga poder a los grandes centros de investigación y revistas de alto impacto que destinan gran parte de sus esfuerzos a estrategias de comunicación.

La homogeneización y selectividad que caracterizaba a la cobertura de la ciencia en la prensa escrita se hace aún más patente en las ediciones digitales

El futuro pasa por trabajar una información plural y contrastada, no dependiente de notas de prensa y que garantice la visibilización no sólo de la ciencia internacional, sino también y con mayor ahínco de la española. En este sentido, nos gustaría destacar que a la fecha en la que escribimos estas conclusiones, 25 de junio de 2015, la edición digital del diario El país ha creado una subsección bajo el paraguas del área de ciencia bajo el título Ciencia en español. Ofrece cobertura de las investigaciones desarrolladas en España y América Latina.

Todos los datos extraídos en el estudio señalan que las ediciones digitales analizadas están proyectando una imagen distorsionada de cómo es el sistema de I+D+i en nuestro país, que no sólo afecta a la promoción de la cultura científica, sino también al apoyo ciudadano a la ciencia. Y si vamos más allá, podríamos llegar a pensar que podría afectar incluso al fomento de vocaciones, al presentar la ciencia como algo lejano de nuestra realidad inmediata, sólo con relevancia si va acompañada del término "internacional".

\section{Bibliografía}

Aranes-Usandizaga, José-Ignacio; Landa-Montenegro, Carmelo (2002). "Periodismo científico: conceptualización y líneas de investigación". Mediatika: Cuadernos de medios de comunicación, n. 8, pp. 293-319.

http://www.euskomedia.org/PDFAnlt/mediatika/08/08293319. $p d f$

Bauer, Martin (1998). "The medicalization of science news from the 'rocket-scalpel' to the 'genemeteorite' complex". Social science information, v. 37, n. 4, pp. 731-751. http://dx.doi.org/10.1177/053901898037004009

Bourdieu, Pierre (2003). El oficio de científico. Ciencia de la ciencia y reflexividad. Barcelona: Anagrama. ISBN: 9788433961983

Brossard, Dominique; Scheufele, Dietram (2013). "Science, new media and the public". Science, v. 339, n. 6115, pp. 40-41. http://dx.doi.org/10.1126/science.1232329

Bucchi, Massimiano; Mazzolini, Renato (2003). "Big science, little news: science coverage in the Italian daily press, 1946
1997". Public understanding science, v. 12, n. 1 pp. 7-24. http://dx.doi.org/10.1177/0963662503012001413

Commission of the European Communities (2006). White paper on a European communication policy. Brussels: Commission of the European Communities.

http://europa.eu/documents/comm/white_papers/pdf/ com2006_35_en.pdf

Comscore (2012). "El mercado online español en un vistazo. Noviembre 2012". ComScore, 20 diciembre.

http://www.comscore.com/esl/Panorama_Digital/Datos_actuales/ El_mercado_online_espanol_en_un_vistazo_- Noviembre_2012

Comscore (2014). "El mercado online español en un vistazo. Diciembre 2013". ComScore, 27 enero.

http://www.comscore.com/esl/Panorama-Digital/Datos-actuales/ El-mercado-online-espanol-de-un-vistazo-Diciembre-2013

Comscore (2014). "El mercado online español en un vistazo. Enero 2014". ComScore, 25 febrero.

http://www.comscore.com/esl/Panorama-Digital/Datosactuales/El-mercado-online-espanol-de-un-vistazo-Enero-2014

Cortiñas-Rovira, Sergi; Ramon-Vegas, Xavier (2013). “Estrategias de difusión de una revista científica. Un experimento con El profesional de la información". El profesional de la información, v. 22, n. 5, pp. 405-414.

http://dx.doi.org/10.3145/epi.2013.sep.05

CSIC (2012). Áreas científicas.

http://www.csic.es/areas-cientificas

Curtin, Patricia; Rhodenbaugh, Eric (2001). "Building the news media agenda on the environment: A comparison of public relations and journalistic sources". Public relations review, n. 27, v. 2, pp. 179-195.

http://dx.doi.org/10.1016/S0363-8111(01)00079-0

De-Semir, Vladimir (2000). "Periodismo científico, un discurso a la deriva". Revista iberoamericana de discurso y sociedad, v. 2, n. 2, pp. 9-37.

De-Semir, Vladimir (2013). "Protagonistas y públicos de la comunicación científica". En: El científico ante los medios de comunicación. Retos y herramientas para una cooperación fructifera. Madrid: Cuadernos de la Fundación Dr. Antonio Esteve, n. 28, pp. 1-8.

De-Semir, Vladimir; Ribas, Cristina; Revuelta, Gema (1998). "Press releases of science journal articles and subsequent newspaper stories on the same topic". JAMA (Journal of the American Medical Association),v. 280, n. 3, pp. 294-295.

http://dx.doi.org/10.1001/jama.280.3.294

Einsiedel, Edna (1992). "Framing science and technology in the Canadian press". Public understanding of science, v. 1, n. 1, pp. 89-101.

http://dx.doi.org/10.1088/0963-6625/1/1/011

Elías, Carlos (2002a). "Influencia de la revistas de impacto en el periodismo científico y en la ciencia actual". Revista española de investigaciones sociológicas, n. 98, pp. 123-137. http://www.reis.cis.es/REIS/PDF/REIS_098_09.pdf

Elías, Carlos (2002b). “Periodistas especializados en ciencia: 
formación, reconocimiento e influencia". Mediatika, n. 8, pp. 389-403.

http://www.euskomedia.org/PDFAnlt/mediatika/08/08389403. $p d f$

Elías, Carlos (2002c). "La revista Nature en las noticias de prensa". Comunicar, v. 10, n. 9, pp. 37-41. http://e-archivo.uc3m.es/handle/10016/19549

Entwistle, Vikki (1995). "Reporting research in medical journals and newspapers". British medical journal, v. 310, n. 6984, pp. 920-923.

http://dx.doi.org/10.1136/bmj.310.6984.920

Fecyt (2013). Percepción social de la ciencia y la tecnología 2012. Madrid: Fundación Española para la Ciencia y la Tecnología.

Fernández-Muerza, Álex (2005). "La información científica en la prensa de referencia: el caso español a partir de un análisis comparativo". Zer, v. 19, pp. 205-232.

http://www.ehu.eus/zer/hemeroteca/pdfs/zer19-10-fernandez. $p d f$

Gans, Herbert (2010). "News and the news media in the digital age: Implications for democracy". Daedalus, v. 139, n. 2, pp. 8-17.

http://dx.doi.org/10.1162/daed.2010.139.2.8

Goirena, Juan-José; Garea, María-Gloria (2002). "La salud de la información científica". Mediatika, v. 8, pp. 523558.

http://www.euskomedia.org/PDFAnlt/mediatika/08/08523558. $p d f$

González-Alcaide, Gregorio; Valderrama-Zurián, Juan-Carlos; Aleixandre-Benavent, Rafael (2009). "La investigación sobre la divulgación de la ciencia en España: situación actual y retos para el futuro". Arbor ciencia, pensamiento y cultura, v. 185, n. 738 , pp. 861-869.

http://dx.doi.org/10.3989/arbor.2009.738n1058

Granado, Antonio (2011). "Slaves to journals, serfs to the web: The use of the internet in newsgathering among European science journalists". Journalism, v. 12, n. 7, pp. 794813.

http://dx.doi.org/10.1177/1464884911412702

Hansen, Anders (1994). "Journalistic practices and science reporting in the British press". Public understanding science, v. 2, n. 3, pp. 111-134.

http://dx.doi.org/10.1088/0963-6625/3/2/001

Hotz, Robert (2002). "The difficulty of finding impartial sources in science". Nieman reports, v. 56, n. 3, pp. 6-7.

http://niemanreports.org/articles/the-difficulty-of-findingimpartial-sources-in-science

Hu, Yifeng; Sundar, Shyam (2010). "Effects of online health sources on credibility and behavioral intentions". Communication research, v. 37, n. 1, pp. 105-132.

http://dx.doi.org/10.1177/0093650209351512

Jerome, Fred (1986). "Prospect for sience journalism". En: Goldstein, Jeffrey (ed.). Reporting science: The case of aggression, pp. 147-154. ISBN: 9780898596083
Kiernan, Vincent (2000). "Mars meteorite: A case study in controls of dissemination of science news". En: Annual meeting of the Association for Education in Journalism and Mass Communication (Baltimore).

http://dx.doi.org/10.1088/0963-6625/9/1/302

López-Pérez, Lourdes; Olvera-Lobo, María-Dolores (2015). "Comunicación de la ciencia 2.0 en España: El papel de los centros públicos de investigación y de medios digitales". Revista mediterránea de comunicación, v. 6, n. 2. http://dx.doi.org/10.14198/MEDCOM2015.6.2.08

Olvera-Lobo, María-Dolores; López-Pérez, Lourdes (2013). "The role of public universities and the primary digital national newspapers in the dissemination of Spanish science through the internet and web 2.0". En: TEEM '13 Procs of the first int conf on technological ecosystem for enhancing multiculturality. New York: ACM, pp. 191-196.

http://dx.doi.org/10.1145/2536536.2536565

Olvera-Lobo, María-Dolores; López-Pérez, Lourdes (2014). "Science communication 2.0: The situation of Spain through its public universities and the most widely-circulated online newspapers". Information resources management journal, v. 27, n. 3 , pp. $42-58$.

http://dx.doi.org/10.4018/irmj.2014070104

Pellechia, Marianne (1997). "Trends in science coverage: A content analysis of three US newspapers". Public understanding of science, v. 6, n. 1, pp. 49-68.

http://dx.doi.org/10.1088/0963-6625/6/1/004

Pérez-Bahón, Félix (2010). "Reflejo del uso de internet como fuente en la información de salud. Análisis de los principales diarios generalistas españoles". Textual \& visual media, n. 3, p. 167-198.

http://textualvisualmedia.com/images/revistas/03/ Reflejo\%20del\%20uso.pdf

Pew Research Center (2006). The state of news media 2006. http://www.journalism.org/topics/state-of-the-newsmedia/2006

Pont-Sorribes, Carles; Cortiñas-Rovira, Sergi; Di-Bonito, Ilaria (2013). "Retos y oportunidades para los periodistas científicos en la adopción de las nuevas tecnologías: el caso de España". Journal of science communication, v. 12, n. 3, pp. 1-17.

http://jcom.sissa.it/sites/default/files/documents/ JCOM1203(2013)A05_es.pdf

Ribas, Cristina (1998). "La influencia de los press release, según con el cristal con el que se mire". Quark, n. 10, pp. 32-37. http://quark.prbb.org/10/articulos.htm

Russell, Cristine (2008). "Science reporting by press release. An old problema grows worse in the digital age". Columbia journalism review.

http://www.cjr.org/the_observatory/science_reporting_ by_press_rel.php?page $=$ all

Stryker, Jo (2002). "Reporting medical information: Effects of press releases and newsworthiness on medical journal articles' visibility in the news media". Preventive medicine, v. 35, n. 5 , pp. $519-530$.

http://dx.doi.org/10.1006/pmed.2002.1102 
Van Trigt, Anke; De-Jong-Van-den-Berg, Lolkje; HaaijerRuskamp, Flora; Willems, Jaap; Tromp, Theo (1994). "Journalists and their sources of ideas and information on medicines". Social science \& medicine, v. 38, n. 4, pp. 637643.

http://dx.doi.org/10.1016/0277-9536(94)90261-5

Veneu, Fernanda; Amorim, Luis-Henrique; Massarani, Luisa (2008). "Science journalism in Latin America: How the scientific information from a scientific source is accommodated into a journalistic story". Journal of science communication, v. 7, n. 1, pp. 1-9.
http://jcom.sissa.it/sites/default/files/documents/Jcom0701(2008)A03.pdf

Weitkamp, Emma (2003). "British newspapers privilege health and medicine topics over other science news". Public relations review, v. 29, n. 3, pp. 321-333. http://dx.doi.org/10.1016/S0363-8111(03)00041-9

Williams, Andy; Clifford, Sadie (2009). Mapping the field: A political economic account of specialist science news journalism in the UK national media. Cardiff University.

http://www.cardiff.ac.uk/jomec/resources/Mapping_ Science_Journalism_Final_Report_2003-11-09.pdf

\title{
Nueva colección EPI Scholar
}

\section{Libros académicos y científicos de Información y Documentación}

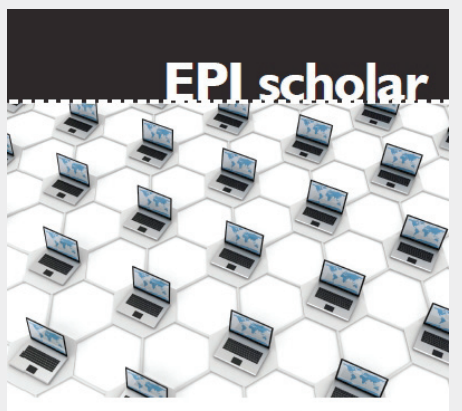

\section{LA WEB SOCIAL} COMO NUEVO MEDIO DE COMUNICACIÓN Y EVALUACIÓN CIENTÍFICA

AWALA MAS-BLEDA ISIDRO F. AGUILLO

\section{La web social como nuevo medio de comunicación y evaluación científica de Amalia Mas-Bleda e Isidro F. Aguillo}

Este libro quiere ser, para los académicos, una guía para difundir mejor sus productos; para los profesionales de la información y quienes trabajan en evaluación científica, un catálogo de altmetría y una ayuda sobre nuevas fuentes y métricas; y para el público en general, un lugar donde encontrar nuevos canales de acceso al conocimiento científico.

Mas-Bleda, Amalia; Aguillo, Isidro F. (2014). La web social como nuevo medio de comunicación y evaluación científica. Barcelona: El profesional de la información, Editorial UOC, 208 pp. ISBN: 9788490649220

\section{SEO. Introducción a la disciplina del posicionamiento en buscadores Jorge Serrano-Cobos}

¿EI SEO es fácil? ¿Difícil? Todo depende. Este libro aporta pistas para desarrollar una estrategia de acciones tácticas que lleven a un posicionamiento estable y ético desde cero en los motores de búsqueda de Internet, sin miedo a los cambios del algoritmo, para aquellos que no saben o que necesitan entender de optimización y posicionamiento en buscadores para coordinarse con otros profesionales. .

Serrano-Cobos, Jorge (2015). SEO. Introducción a la disciplina del posicionamiento en buscadores. Barcelona: El profesional de la información, Editorial UOC, 202 pp. ISBN: 978849064 9565

\author{
Información: Isabel Olea \\ epi.iolea@gmail.com
}

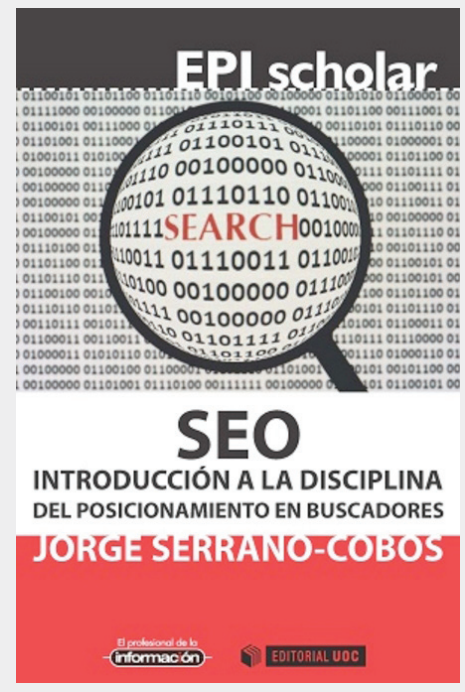

http://www.elprofesionaldelainformacion.com/librosEPIScholar.html 